\title{
ANCA-Associated Vasculitis in a Man with Scleroderma and Pulmonary Fibrosis
}

\author{
Alyson Wong MD FRCPC, Christine McDonald MD FRCPC, John Thenganatt MD FRCPC
}

DOI: $10.22374 /$ cjgim.v13i3.164

\begin{abstract}
About the Authors Hospital, Canada.

Corresponding author: john.thenganatt@uhn.ca.

Submitted: January 16, 2017; Accepted: April 8 2018. Published: August 27, 2018.
\end{abstract}

Alyson Wong is with the Division of Respirology, Department of Medicine, Dalhousie University, Halifax, Canada. Christine McDonald is with the Division of Respirology, Sunnybrook Health Sciences Centre, Department of Medicine, University of Toronto, Toronto, Ontario, Canada. John Thenganatt are with the Division of Respirology, Department of Medicine, University Health Network, Toronto General

\begin{abstract}
Systemic sclerosis is a connective tissue disease that is characterized by small-vessel vasculopathy, production of autoantibodies and fibroblast dysfunction resulting in increased deposition of extracellular matrix. It typically causes fibrosis of the skin and potentially internal organs. The most common lung findings in those with systemic sclerosis are interstitial lung disease and pulmonary hypertension. Here we describe a 58-year-old man with systemic sclerosis, interstitial lung disease and pulmonary arterial hypertension. He presented with cough and dyspnea and had diffuse micronodules on chest imaging. He also had new and progressive renal impairment that led to a renal biopsy revealing an ANCA-associated vasculitis.
\end{abstract}

\section{RESUME}

La sclérose systémique est une maladie du tissu conjonctif caractérisée par une vasculopathie des petits vaisseaux, la production d'auto-anticorps et un dysfonctionnement des fibroblastes entraînant une augmentation du dépôt de la matrice extracellulaire. Elle provoque généralement une fibrose de la peau et potentiellement des organes internes. Les résultats pulmonaires les plus courants chez les personnes atteintes de sclérodermie systémique sont la maladie pulmonaire interstitielle et l'hypertension pulmonaire. Nous décrivons ici un homme de 58 ans souffrant de sclérodermie systémique, de maladie pulmonaire interstitielle et d'hypertension artérielle pulmonaire. Il présentait une toux et une dyspnée et avait des micronodules diffus sur l'imagerie thoracique. Il a également eu une nouvelle insuffisance rénale progressive qui a conduit à une biopsie rénale révélant une vascularite associée aux ANCA.

\section{Case Summary}

A 58-year-old man presented to hospital with a one-month history of shortness of breath on exertion, weight loss and nonproductive cough with no hemoptysis. His past medical history was significant for systemic sclerosis manifesting as Raynaud's phenomenon, puffy fingers, telangiectasia and abnormal nail fold capillaries. His extractable nuclear antigen panel was negative for autoantibodies at the time of diagnosis. He also had interstitial lung disease and pulmonary arterial hypertension
(PAH). The PAH was treated with Bosentan and Tadalafil. His mild interstitial lung disease did not require therapy. Computed tomography (CT) of the thorax from 3 years prior to admission is shown in Figure 1.

On examination, he was afebrile and hypoxic with an oxygen saturation of $75 \%$ breathing ambient air. His blood pressure was $125 / 71 \mathrm{mmHg}$ and pulse rate was 91 beats per minute (regular). There were bibasilar inspiratory crackles. Cardiac examination revealed a loud P2. There were no signs of volume overload. 
Bloodwork was significant for a white blood cell count of $13,100 / \mu \mathrm{L}$ and an elevated creatinine of $138 \mu \mathrm{mol} / \mathrm{L}$ (normal at baseline). Computed tomography of the thorax revealed new bilateral pulmonary micronodules in a random pattern, bibasilar consolidation, and mediastinal and hilar lymphadenopathy (Figure 2).

The patient was admitted to hospital and treated for suspected community-acquired pneumonia. However, his symptoms and hypoxia did not improve on antibiotics. A bronchoscopy and bronchoalveolar lavage (BAL) was performed to identify a potential pathogen; endobronchial ultrasound-guided transbronchial needle aspiration (EBUS-TBNA). was performed to sample the mediastinal lymphadenopathy for cytology and microbiology. BAL fluid returned positive for parainfluenza type 1. TBNA culture and AFB were negative; TBNA cytology revealed benign lymphoid tissue.

Over the course of his hospitalization, his renal function progressively declined. His urinalysis was positive for greater than 200 red blood cells per $\mu \mathrm{L}$ and $3 \mathrm{~g} / \mathrm{L}$ of protein. Urine microscopy showed white blood cell casts. Given the worsening renal function and active urine sediment, further testing for glomerulonephritis was performed. Serology was positive for anti-neutrophil cytoplasmic antibodies with anti-MPO antibodies $>8$ units $/ \mathrm{mL}$. ESR and CRP were elevated at $113 \mathrm{~mm} / \mathrm{h}$ and 129 $\mathrm{mg} / \mathrm{L}$, respectively. Serology for hepatitis B and C were negative. Repeat serologic testing revealed the presence of anti-SS-A and anti-RNP antibodies. Rheumatoid factor was positive at $92 \mathrm{IU} /$ $\mathrm{mL}$ and anti-CCP was negative. The patient's kidney function continued to deteriorate with a peak creatinine of $254 \mu \mathrm{mol} / \mathrm{L}$. He went on to have a kidney biopsy that showed pauci-immune necrotizing crescentic glomerulonephritis. A diagnosis of ANCAassociated vasculitis was made.

He was treated with 1 gram of intravenous methylprednisolone for 3 days, followed by $60 \mathrm{mg}$ of prednisone daily with improvement in serum creatinine. Oral cyclophosphamide (50 $\mathrm{mg}$ daily) was added. It was only after the initiation of steroids, that his shortness

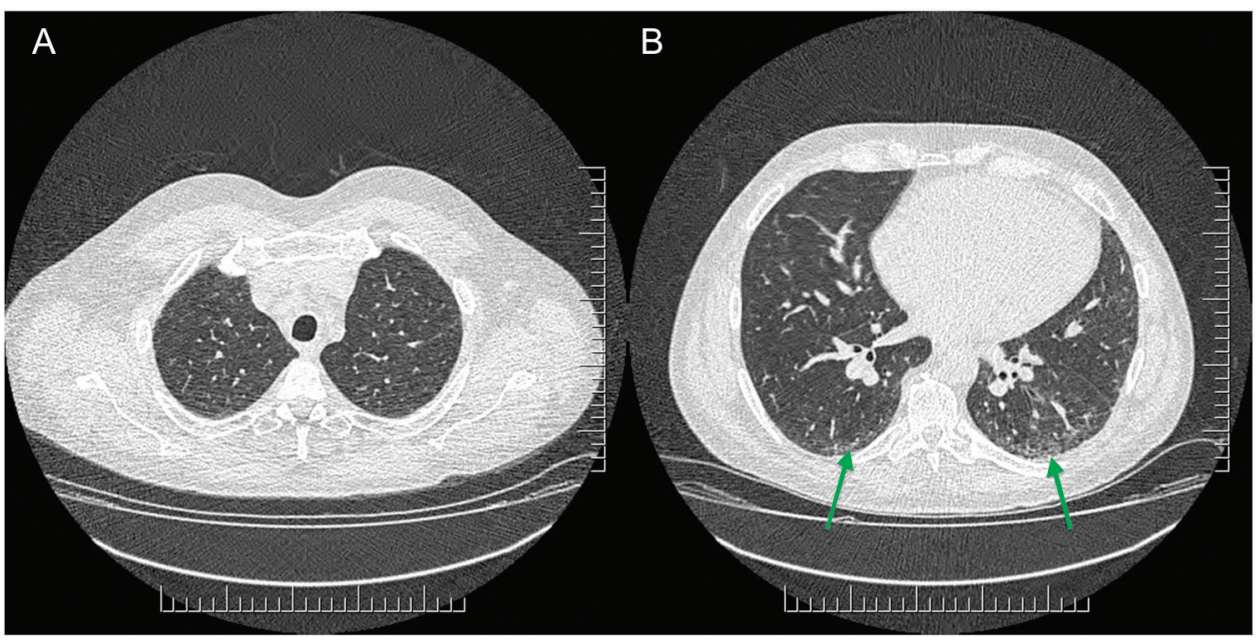

Figure 1. Computed tomography of thorax. ( $A$ and B) 2012. Three-years prior to presentation, the patient had reticulation at the bases bilaterally (green arrows), however no nodules or mediastinal lymphadenopathy.

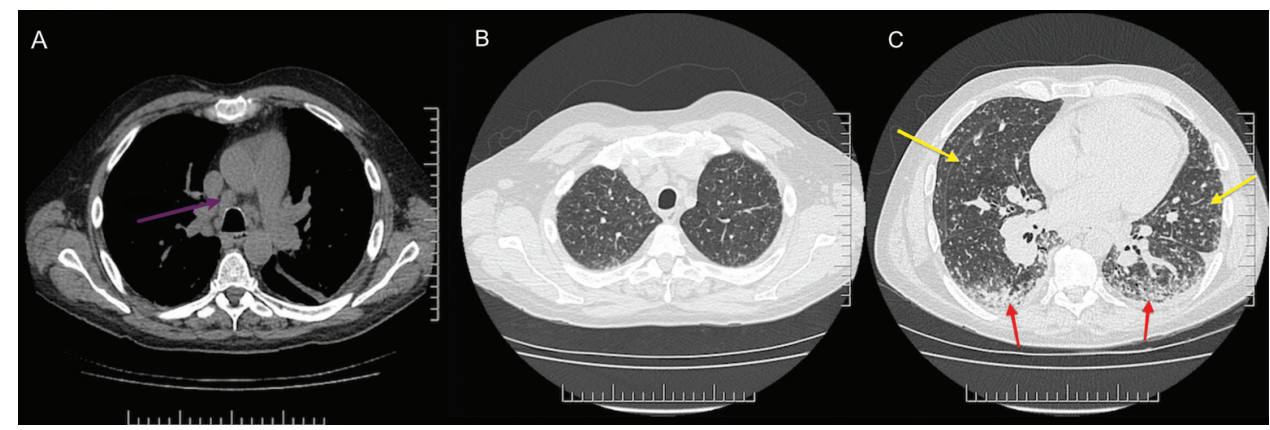

Figure 2. Computed tomography of thorax on admission. (A, B and C) March 2015. There were new bilateral pulmonary micronodules (yellow arrow) in a random pattern, bibasilar consolidation (red arrows) and mediastinal and hilar lymphadenopathy (purple arrow). 


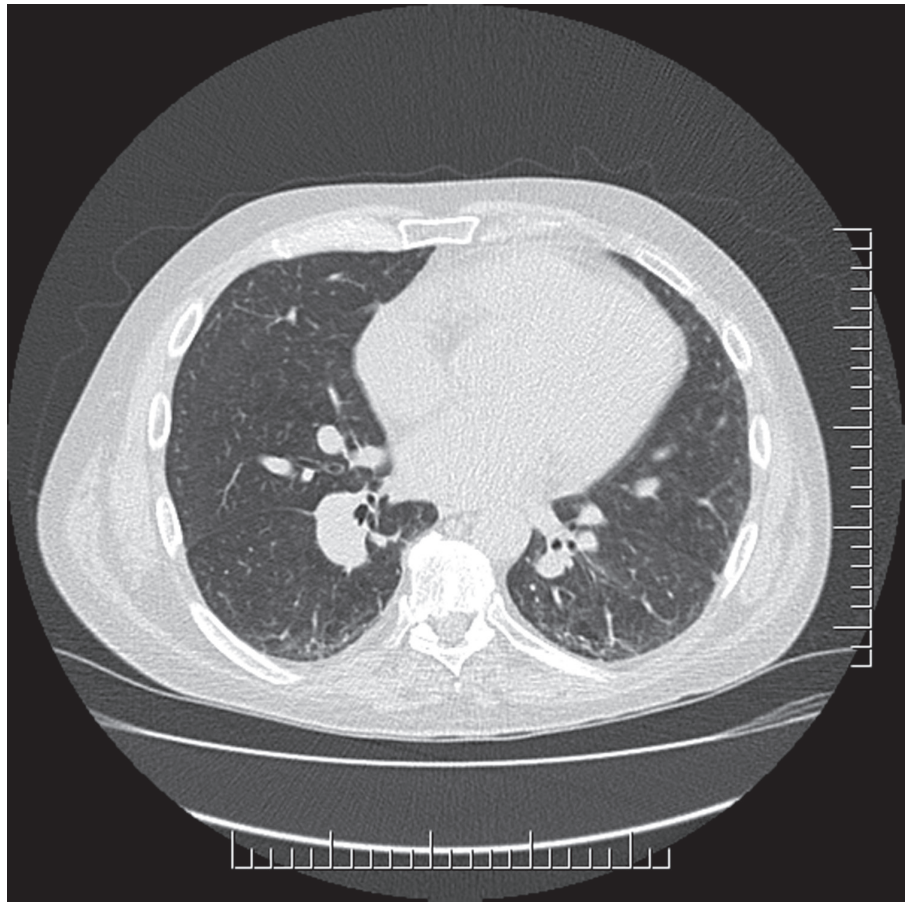

Figure 3. Follow-up computed tomography of thorax, June 2015. Two months after receiving treatment for ANCA-associated vasculitis with steroids and cyclophosphamide, the micronodules and mediastinal lymphadenopathy had resolved.

of breath and oxygen requirements significantly decreased. At the time of hospital discharge, the patient required oxygen on exertion only and his creatinine was $168 \mu \mathrm{mol} / \mathrm{L}$. His follow-up CT thorax 2 months later demonstrated marked improvement in micronodules, consolidation and mediastinal lymphadenopathy (Figure 3).

\section{Discussion}

ANCA-associated vasculitis (AAV) is an inflammatory disease of small to medium sized blood vessels characterized by the presence of ANCA that specifically target proteinase-3 (PR3) or myeloperoxidase (MPO) antigens within the cytoplasm of neutrophils. ${ }^{1-3}$ Granulomatosis with polyangiitis (GPA), microscopic polyangiitis (MPA) and eosinophilic granulomatosis (EGPA) comprise the small-vessel vasculitides that are associated with the presence of ANCA. ${ }^{4}$ The antibody to MPO is more commonly associated with MPA (30-80\%) and EGPA (30-75\%), compared to GPA (0-10\%). ${ }^{4}$ Microscopic polyangiitis shows no clinical or pathologic evidence of granulomatous inflammation, whereas EGPA can have granulomatous nodules in the lung and is characterized by asthma and eosinophilic lung infiltrates. ${ }^{4}$ Our patient did not have asthma or other clinical features of EGPA, making the diagnosis less likely. The clinical features of
MPA include weeks to months of constitutional symptoms such as fatigue, fever and arthralgias, followed by development of renal disease, typically rapidly progressive glomerulonephritis. ${ }^{5}$

The most common radiographic findings for AAV consist of nodules, masses or consolidation often with cavitation. ${ }^{6}$ Given the rapid improvement in our patient's respiratory status, we believe that the chest imaging findings were likely related to AAV and not solely due to parainfluenza virus. The only BAL performed in our patient was before the diagnosis of AAV was considered. Therefore, we were unable to confirm the presence of alveolar hemorrhage. Once ANCA-associated vasculitis was diagnosed, a repeat bronchoscopy was not performed to assess for alveolar hemorrhage as it would not have altered our management. An open lung biopsy was not performed due to high risk of bleeding in patients with underlying pulmonary hypertension.

The overlap of systemic sclerosis (SSc) and AAV is rare. A prior study examined a database of 2,200 patients with scleroderma and found 35 patients had evidence of vasculitis with only 10 of these patients being ANCA positive $(0.4 \%$ of all patients). ${ }^{7}$ These patients with AAV had a higher tendency to have anti-MPO antibodies compared to anti-PR3 antibodies ( $87.5 \%$ vs. $12.5 \%) .^{7}$ Interestingly, the anti-RNP antibody (which was positive in our patient) was found to be associated with SSc/ AAV overlap. ${ }^{7}$ However, the antibodies that are specific for the diagnosis of systemic sclerosis (centromere and Scl-70) were not associated with SSc/AAV overlap. ${ }^{7}$ The clinical presentation of these patients with overlap can be varied with some patients having few features of vasculitis. ${ }^{7}$ There is also an increased occurrence of pulmonary fibrosis in these patients compared to those who have either scleroderma or AAV alone. ${ }^{7}$ Pulmonary fibrosis was present in $80 \%$ of SSc/AAV overlap patients compared to $7 \%$ of patients with MPA only. ${ }^{7,8}$

\section{Conclusion}

Although a rare combination, having systemic sclerosis may increase the risk of developing AAV and it is important to consider given the potential life-threatening outcome of missing such a diagnosis. If AAV is left untreated, the mortality rate is approximately $82 \%$ within the first year after diagnosis, most commonly due to rapidly progressive renal failure and respiratory failure. ${ }^{9}$ Further understanding of how systemic sclerosis and AAV are connected is required.

\section{Source(s) of Support}

None.

\section{Conflict of Interest Statements}

The authors declare that there are no conflicts of interest. 


\section{References}

1. van den Hoogen F, Khanna D, Fransen J, et al. Classification Criteria for Systemic Sclerosis: an American College of Rheumatology/European League against Rheumatism Collaborative Initiative. Arthrit Rheumatis 2013;65(11):2737-47.

2. Strollo D, Goldin J. Imaging lung disease in systemic sclerosis. Curr Rheumatol Rep 2010;12:156-61.

3. Kaldas A, Warraich I, Prabhakar S. ANCA associated glomerulonephritis- an in-depth review. J Nephrol Ther 2013;4:147-59.

4. Jose A. Gómez-Puerta JA, Hernández-Rodríguez J, López-Soto A, et al. Antineutrophil cytoplasmic antibody-associated vasculitides and respiratory disease. Chest 2009;136(4):1101-11.
5. Brown K, Frankel S, Cool C. Murray and Nadel's Textbook of Respiratory Medicine. Elsevier, 2016.

6. Chung MP, Yi CA, Lee HY, Han J, Lee KS. Imaging of pulmonary vasculitis. Radiology 2010;255(2):322-41.

7. Derrett-Smith EC, Nihtyanova SI, Harvey J, Salama AD, Denton CP. Revisiting ANCA-associated vasculitis in systemic sclerosis: clinical, serological and immunogenetic factors. Rheumatology 2013;52(10):1824-31.

8. Arulkumaran N, Perielneris N, Gaskin G, et al. Interstitial lung disease and ANCA-associated vasculitis: a retrospective observational cohort study. Rheumatology 2011;50:1035-43.

9. Berden A, Goceroglu A, Jayne D, et al. Diagnosis and management of ANCA associated vasculitis. BMJ 2012;344:e26. 